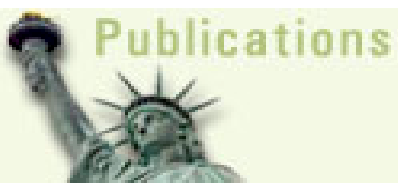

American Journal of Biochemistry and Biotechnology 3 (4): 225-232, 2007

ISSN 1553-3468

(C) 2007 Science Publications

\title{
Redox Sensing and Cardiac Myocyte Loss: Some Recent Insights
}

\author{
Shyamal K. Goswami \\ School of Life Sciences, Jawaharlal Nehru University, New Delhi 1100667, India
}

\begin{abstract}
Generation of reactive oxygen-nitrogen species (ROS/RNS) has long been ascribed to pathophysiological conditions like ageing, neurodegenerative and cardiovascular disorders. However, more recent evidences suggest that while generation of ROS/RNS at a higher threshold is deleterious, at a lower dose these can act as second messengers, influencing specific cellular events like cell growth and differentiation, embryonic development, protection against ischemic injuries and progression of diseases like cancer. With the advent of sophisticated tools of biochemistry and cell biology, it is becoming apparent that various ROS/RNS, when generated transiently at a moderate level, can reversibly modify proteins, causing loss-gain of functions, analogous to that by phosphorylationdephosphorylation. More significantly, recent studies also suggest that even apoptosis induced by ROS/RNS might also involve discrete cell signaling rather than general surge of ROS/RNS as the dterminant. Taken together, coming days are likely to reveal more discrete nature of ROS/RNS signaling in various cellular contexts including cell death.
\end{abstract}

Key words: Reactive oxygen-nitrogen species, Cell signaling, Redox-signaling, Cell survival, Apoptosis.

Intracellular generation of free radicals and/or reactive oxygen species has long been attributed to deleterious effects such as cell death (apoptosis), ageing, neurodegenerative and cardiovascular diseases $[1,2]$. The general term used by the biologists in describing these phenomena is "oxidative stress"; first coined by Nakashima et. al in late seventies and since it has been one of most widely investigated subject in biology with 45930 entries in Pubmed as on February 9, $2007^{[3]}$. Interestingly, in spite of extensive research for about thirty years, understanding of mechanisms of oxidative stress and its effects on cellular processes have been painstakingly evolving ${ }^{[1,2,4-6]}$. Noticeably, a large number of experimental evidences, especially during the past decade, have suggested that although the generation of reactive oxygen/nitrogen species at a higher threshold is deleterious, at a lower dose these can act as second messengers, transmitting intracellular signals (as done by kinases and phosphatases), regulating specific cellular events like cell growth and differentiation, embryonic development, protection against ischemic injuries and diseases like cancer ${ }^{[5-8]}$. With the advent of sophisticated tools of biophysical chemistry, a plethora of free radicals and reactive oxygen species have been identified till date and their effects on cellular macromolecules have been investigated ${ }^{[9]}$. Furthermore, following the seminal discovery of nitric oxide as intracellular messenger ${ }^{[10]}$, it was appreciated that in the intracellular milieu, generation of reactive oxygen species are highly interlinked with that of a number of reactive nitrogen species and thus the biology of reactive oxygen/nitrogen species are often investigated in conjunction. As an example, nitric oxide can interact with superoxide radical (O2.), hydrogen peroxide $(\mathrm{H} 2 \mathrm{O} 2)$, or transition metal centers resulting in the generation of a number of highly reactive entities ${ }^{[9]}$. Although reactive oxygen species have long been known for their deleterious effects on cellular macromolecules (proteins, DNA and lipids); accumulating evidences suggest that reactive oxygen/nitrogen species, especially when generated in limited amounts in a specific location (intracellular), can reversibly modify certain proteins and modulate their functions, thereby acting as second messengers [11]. The present review is aimed towards describing some of the recent studies related to the role of reactive oxygen/nitrogen species (ROS/RNS) in cell signaling in general and in modulating cell death and survival in particular.

Reactive oxygen/nitrogen species (ROS/RNS) and their roles in biology: Free radicals contain one or more unpaired electrons in the outermost orbital of their constituent atoms, making them highly reactive and

Corresponding Author: $\quad$ Shyamal K. Goswami, School of Life Sciences, Jawaharlal Nehru University, New Delhi 1100667, India 
labile (vis-à-vis their normal counterparts carrying paired electrons; 9, 11, 12). Certain other compounds like hydrogen peroxide, is not a free radical as such; but in presence of metal ions it generates hydroxyl radicals $(\mathrm{OH}$.$) , and thus in a specific context, \mathrm{H} 2 \mathrm{O} 2$ is a reactive (oxygen) species ${ }^{[9,11,12]}$. While certain pathological stimuli generate ROS/RNS with deleterious consequences; certain others like cytokines and growth factors, environmental toxicants like tobacco smoke, ultra-violet radiation, sphingolipids, etc induce ROS/RNS as a physiological response with distinctive effects ${ }^{[5-8,13-15]}$. The effects of ROS/RNS can also be context and tissue specific. While in phagocytic cells, superoxide free radicals (O2.-) generated by nicotinamide adenine dinucleotide phosphate (NADPH)-oxidase complex kill invading microbes in the phagosomes; in fibroblasts, endothelial cells etc., O2.- is generated by NADPH-oxidase at a lower and constitutive level that is involved in cell signaling ${ }^{[16,17]}$. Alike reactive oxygen species, a number of nitrogen containing free radicals and reactive species (RNS) are also generated under various pathophysiological conditions and nitric oxide synthase (both inducible and constitutive) play a nodal role in this process ${ }^{[9,18]}$.

Intracellular generation of ROS/RNS and redox homeostasis: Mitochondria, being the key mediator of oxidative metabolism, are a major source of intracellular ROS ${ }^{[19]}$. Mitochondrial electron transport chain (complex I to IV) is a highly structured assembly of redox-proteins dedicated to the generation of a proton gradient across the inner mitochondrial membrane that is utilized for ATP synthesis. Under decreased ATP requirement, electron transport is inhibited by a feedback mechanism; leading to an accumulation of electrons in the transport chain which then leak out, bind to oxygen and generate O2.-. Amongst four electron transport complexes, complex I and III are the primary producer of $\mathrm{O} 2$. - $^{[20,21]}$. Since O2.- is a highly reactive free radical, it is instantaneously attenuated into less reactive hydrogen peroxide by superoxide dismutase (Mn-SOD in the mitochondria and $\mathrm{Cu} / \mathrm{Zn}-\mathrm{SOD}$ in the cytosol]. $\mathrm{H} 2 \mathrm{O} 2$ is then converted into oxygen and water by catalase. However, if generated in excess, O2.- may escape this attenuation process producing a number of other ROS/RNS through various intracellular pathways ${ }^{[22]}$. As an example, O2.- reacts with NO., generating peroxynitrite (ONOO-). This reaction is diffusion controlled and is of potential importance in macrophages and endothelial cells which simultaneously produce O2.- and NO.. Peroxynitrite is highly reactive and interacts with lipids, DNA, and proteins. When it is of low intensity, it brings in subtle modifications of cellular proteins, inducing cell signaling; while upon high intensity, it causes extensive oxidative injury resulting in cell death by necrosis and apoptosis [23]. Peroxynitrite generation has been attributed to numerous pathological conditions like stroke, myocardial infarction, heart failure, diabetes, circulatory shock, chronic inflammatory diseases, cancer, and neurodegenerative disorders ${ }^{[24]}$. Also, upon protonation, peroxynitrite produces $\mathrm{ONOOH}$, which then dissociates into either NO2. (nitrogen dioxide radical) and $\mathrm{OH}$. or $\mathrm{NO} 3-$ and $\mathrm{H}+{ }^{[9]}$. In addition to mitochondria, several other organelles like microsomes (cycloxigenase) and perxisomes (xanthine oxidase) may also contribute towards the intracellular pool of ROS ${ }^{[25}$, ${ }^{26]}$. Other reactive species such as nitroxyl radical (HNO), especially in the context of ischemic brain, have also been described ${ }^{[27]}$.

Although the side chains of a number of amino acids are responsive to oxidative and nitrosative modifications (by ROS/RNS), cysteine thiols are most sensitive amongst them and thus have drawn have drawn considerable attention ${ }^{[28]}$. Many of the cysteine oxidations are reversible, transiently activating/ inactivating the respective proteins and thus is functionally analogous to phosphorylation-dephosphorylation ${ }^{[28]}$. Oxidative modifications of cysteines play a critical role in pathobiology in general and cardiovascular biology in particular ${ }^{[29]}$. As expected, nature has thus evolved an elaborate set of enzymes and low molecular weight peptides viz., thioredoxin and glutaredoxin oxidoreductases that play a key role in restoring protein thiols and thereby maintaining redox homeostasis. Noticeably, these proteins are functionally equivalent to free radical scavengers, but with much wider role(s) in regulating cellular metabolism, signaling and gene expression ${ }^{[30,31]}$. Thioredoxin is a general disulphide reductase with a low redox potential and thus capable of acting upon a wide range of oxidized proteins. In mammals, there are two thioredoxins i.e., Trx1 (cytosolic) and Trx2 (mitochondrial) ${ }^{[32]}$. Antioxidant effects of thioredoxin is mediated via its interaction with a diverse family of regulatory molecules like Apoptotic signal regulating kinase 1 (ASK-1), Vitamin D3-upregulated protein-1 (VDUP1), gene regulatory proteins NFkB and AP-1 ${ }^{[33 \text {, }}$ 34]. Thioredoxin has also been attributed to the upregulation of antioxidant genes like Mn-SOD and Hemeoxiganase-1 ${ }^{[35]}$. Like thioredoxin, glutaredoxin system comprising glutaredoxin, glutathione and 
glutathione reductase also play a key role in maintaining intracellular redox equilibrium. In mammals, there are two glutaredoxin genes viz. Grx 1 and Grx $2^{[36]}$. Mitochondrial electron transport chain being a major source of ROS leads to reduced GSH/GSSG ratio and glutathionylation of various proteins in complex I while Grx 2 plays a major role in regenerating key mitochondrial proteins by deglutathionylation ${ }^{[37]}$.

Taken together, generation of various ROS/RNS and their simultaneous attenuation by the antioxidant enzymes may lead to transient and reversible oxidative/nitrosative modifications of cellular proteins which may generate specific signals. Also, excessive generation of ROS/RNS may cause extensive (and non specific) modifications of cellular proteins (such as formation of carbonyls, oxidation of methionines/cysteines and cross-linking of polypeptides), lipids and DNA with deleterious consequences ${ }^{[38-41]}$.

Cell signaling by ROS/RNS: Potential role of a reactive species in cell signaling was revealed by the discovery of nitric oxide as a signal transducer ${ }^{[10]}$. Since, a number of other ROS/RNS have also emerged as the mediators of intracellular signals, resulting in a paradigm shift in redox biology ${ }^{[11]}$. It is now believed that ROS/RNS, when generated for a brief period at a moderate level, can transiently and reversibly modify proteins, causing loss-gain of functions ${ }^{[11,42]}$. Second messengers are characterized by their transitory nature and specificity of action ${ }^{[43]}$. In that context, ROS/RNS are also generated upon receptor stimulation, they are very transient in nature, but their specificity of action (except that of nitric oxide) has often been questioned $[11,44,45]$. As an example, while $\mathrm{O} 2$. and $\mathrm{H} 2 \mathrm{O} 2$ are highly diffusible, less reactive and therefore might act in a specific manner; other highly reactive species like $\mathrm{OH}$. is unlikely to have any specificity ${ }^{[46]}$. Nevertheless, generation of ROS/RNS has been attributed to the modulation of a number of cell signaling/gene regulatory modules including MAP kinase, PI3-kinase/Akt, transcription factors AP-1 and $\mathrm{NF \kappa B}{ }^{[47-49]}$. A few direct targets of oxidative/nitrosative modifications have also identified till date and those are primarily the cysteine residues present in the catalytic sites of the respective proteins $[11,50,51]$. Noticeably, a number of non-signaling regulatory modules like ryanodine receptor, Sarco/endoplasmic $\mathrm{Ca} 2+$ pumps (SERCA) are also targeted by ROS/RNS. Ryanodine receptor contains a large number of cysteine residues and redox- modifications of at least some of those modulate its function ${ }^{[52,53]}$. Nitric oxide (NO) can also form Snitroso derivatives of some of those cysteine residues and thereby play a role in modulating its activity ${ }^{[54]}$. Similarly, peroxynitrite inhibits SERCA activity by oxidative modifications, thereby affecting calcium transients ${ }^{[55]}$.

Redox homeostasis and cell survival: Aerobic organisms harness energy by the oxidation of glucose through a series of redox reactions conserved in nature. A plethora of other redox-sensitive molecules, also conserved during evolution, carry out other cellular functions. Nevertheless, by large, intracellular environment is reducing in nature and excessive generation of ROS/RNS perturbs the redox equilibrium. Whenever ROS/RNS is generated in the cellular milieu, it reacts with lipids, proteins, carbohydrates and nucleic acids and the outcome depends upon the intracellular localization, amplitude and the life span of the reactive species as well as the cell type ${ }^{[56-61]}$. Since redox homeostasis is critical for normal cellular functions, cells also have an extensive network of antioxidant enzymes like heme oxygenase, superoxide dismutase, catalase, glutathione peroxidase, thioredoxinthioredoxin reductase, glutaredoxin-glutaredoxin reductase and small molecules like vitamin $\mathrm{E}$ and $\mathrm{C}^{[62-}$ ${ }^{64]}$. Thus, while an elaborate assembly of pro-oxidant and antioxidant enzymes and other biomolecules generate, utilize and attenuate intracellular ROS/RNS for various biological purposes; any disharmony in this redox-equilibrium has profound effects on the cellular physiology ${ }^{[65-67]}$.

ROS/RNS induce apoptosis in cardiac myocytes by multiple mechanisms: Although the deleterious effects of ROS/RNS on cellular processes have been known for many years; it is only recently, biologists had a better understanding of the specific mechanisms by which reactive species elicit cognate responses. Apoptosis or programmed cell death is one of the major consequences of cellular injuries caused by the generation of RO/RNS. Furthermore, by definition, apoptosis is a genetically programmed conserved sequence of events with distinct characteristics. However, the mechanisms of inducing apoptosis by various stimuli can be diverse and whether a cell will opt for apoptosis or survival depends on the agonist/stimuli as well as the cellular context ${ }^{[68-70]}$. Noticeably, based on the accumulating evidences, Buttke and Sandstrom for the first time suggested in 1994, a unifying hypothesis, according to which 
oxidative stress can be a common mediator of apoptosis induced by various agents ${ }^{[71]}$. In agreement, numerous studies thereafter have attributed ROS/RNS generation towards apoptosis induced by various pathophysiological stimuli ${ }^{[72-75]}$.

The phenomena of apoptosis have been extensively investigated for almost all known cell types from yeast to mammals. However, it has been of intense interest for cardiac myocytes and neuronal cells as these cells are of minimum regenerative potential [70, 76-79]. Neonatal rat cardiac myocytes, upon exposure to various pathophysiological agonists, induce intracellular ROS followed by hypertrophic or apoptotic response depending upon the agonist type and its concentration [80-83]. Attenuation of ROS by pharmacological and molecular inhibitors mitigates these responses ${ }^{[81,83]}$. Also upon treatment with an exogenous oxidant like $\mathrm{H} 2 \mathrm{O} 2$ at low to moderate doses $\left(\begin{array}{ll}\leq 30 & \mathrm{M}\end{array}\right)$ myocytes elicit hypertrophic response while further increase in concentration (up to $200 \quad$ M) leads to apoptosis ${ }^{[83]}$. These studies thereby evolved a conceptual framework according to which a moderate level of ROS generation leads to hypertrophy and at elevation of a yet unspecified threshold, induces apoptosis ${ }^{[84]}$. However, recent study from our laboratory has demonstrated that $\mathrm{H} 9 \mathrm{c} 2$ cardiac myoblasts upon treatment hypertrophic and apoptotic doses of norepinephrine $[2 \mu \mathrm{M}$ and $100 \mu \mathrm{M}$ respectively) induces ROS at comparable levels while eliciting cognate responses ${ }^{[85]}$. Also, as assessed by the induction of a number of downstream mediators like transcription factors AP-1 and Nrf-2, the respective signals were different for hypertrophy and apoptosis, thereby indicating the existence of distinct signaling pathways, in which ROS might be a contribute in a discrete rather than a dose dependent manner ${ }^{[85]}$. In agreement to this observation, Frazier et al., have also demonstrated that in cardiac myocytes upon ischemiareperfusion, ROS is generated from mitochondria; induces a pathway involving proline-rich kinase (Pyk2), the small GTP binding factors Rac-1 and Cdc42, protein kinase-c (PKC) and TGF-beta activated kinase 1 (TAK-1) leading to the activation of JNK ${ }^{[86]}$. In another study, Yaniv et al., have recently demonstrated that cardiac myocytes upon treatment with $\mathrm{H} 2 \mathrm{O} 2$ become sensitized to Fas mediated apoptosis via a Daxx-ASK-1-JNK pathway ${ }^{[87]}$. Taken together, it appears that norepinephrine, ischemia-reperfusion and exogenous oxidant $\mathrm{H} 2 \mathrm{O} 2$, induce $\mathrm{ROS}$ mediated discrete signal pathways in cardiac myocytes leading to cell death. Such prospect of divergence in oxidative signaling is further exemplified by a recent report that in failing heart, loss of myocytes occurs concurrently by three independent mechanisms viz., apoptosis, autophagy and necrosis ${ }^{[88]}$.

\section{CONCLUSION}

Roles of free radicals and other reactive species in biology have been an puzzle for many years. With the advent of sophisticated tools of analytical chemistry, biochemistry, cellular and molecular biology has revealed the existence of various reactive species and their functions in biological processes including cell death, differentiation and survival. However, the precise mechanisms by which they mediate such divergent effects are still emerging. On the contrary to prevailing tenets that a general surge of ROS under various pathophysiological conditions triggers cell death; recent evidences suggest that apoptosis might as well be induced by discrete redox signaling. Thus in coming years, more complex roles ROS in cell death and survival is likely to emerge.

\section{ACKNOWLEDGEMENTS}

This author acknowledges the financial support from the University Grants Commission, India (under the University with Potential for Excellence Program) and the Department of Biotechnology, Govt. of India

\section{REFERENCES}

1. Evans M D., M. Dizdaroglu, M. S. Cooke, 2004. Oxidative DNA damage and disease: induction, repair and significance. Mutat Res, 567(1):1-61

2. Kregel K. C., H. J. Zhang, 2006. An Integrated View of Oxidative Stress in Aging: Basic Mechanisms, Functional Effects and Pathological Considerations. Am J Physiol Regul Integr Comp Physiol, 292(1):R18-36.

3. Nakashima K., K. Yamauchi, S. Miwa, K. Fujimura, A. Mizutani, A. Kuramoto, 1978. Glutathione reductase deficiency in a kindred with hereditary spherocytosis. Am J Hematol, 4(2):141150.

4. Rice-Evans C.A., A.T. Diplock, 1993. Current status of antioxidant therapy. Free Radic Biol Med. 15(1):77-96.

5. Hensley K., K. A. Robinson, S. P. Gabbita, S. Salsman, R. A. Floyd, 2000, Reactive oxygen species, cell signaling, and cell injury. Free Radic Biol Med, 28(10):1456-1462 
6. Mikkelsen R.B., P. Wardman, 2003. Biological chemistry of reactive oxygen and nitrogen and radiation-induced signal transduction mechanisms. Oncogene, 22(37):5734-5754.

7. Esposito F., R. Ammendola, R. Faraonio, T. Russo, F. Cimino. 2004, Redox control of signal transduction, gene expression and cellular senescence. Neurochem Res, 29(3):617-628.

8. Sauer H., M. Wartenberg, 2005, Reactive oxygen species as signaling molecules in cardiovascular differentiation of embryonic stem cells and tumorinduced angiogenesis. Antioxid Redox Signal, 7(11-12):1423-1434.

9. O. Augusto, M. G. Bonini, A. M. Amanso, E. Linares, C. C. Santos, S. L. De Menezes, 2002. Nitrogen dioxide and carbonate radical anion: two emerging radicals in biology. Free Radic Biol Med, 32(9):841-859.

10. Moncada S., R. M. Palmer, E. A. Higgs, 1988. The discovery of nitric oxide as the endogenous nitrovasodilator. Hypertension, 12(4):365-372.

11. Forman H J, Fukuto J M, M.Torres, 2004. Redox signaling: thiol chemistry defines which reactive oxygen and nitrogen species can act as second messengers. Am J Physiol Cell Physiol, 287: C246-C256.

12. Cepinskas G., T. Rui, P. R. Kvietys. 2002. Interaction between reactive oxygen metabolites and nitric oxide in oxidant tolerance. Free Radic Biol Med, 33(4):433-440.

13. Meng T.C., D. A. Buckley, S. Galic, T. Tiganis, N.K. Tonks, 2004. Regulation of insulin signaling through reversible oxidation of the protein-tyrosine phosphatases TC45 and PTP1B. J Biol Chem, 279(36): 37716-37725.

14. Gensch E., M. Gallup, A. Sucher, D. Li, A. Gebremichael, H. Lemjabbar, A. Mengistab, V. Dasari, J. Hotchkiss, J. Harkema, C. Basbaum, 2004. Tobacco smoke control of mucin production in lung cells requires oxygen radicals AP-1 and JNK. J Biol Chem, 279(37):39085-39093.

15. Won JS, I. Singh, 2006. Sphingolipid signaling and redox regulation. Free Radic Biol Med, 40(11):1875-1888.

16. Moreland J.G, A.P. Davis, G. Bailey, W. M. Nauseef, F. S. Lamb, 2006, Anion channels including $\mathrm{CLC}-3$ are required for normal neutrophil oxidative function, phagocytosis, and transendothelial migration. J Biol Chem, 281(18): 12277-12288.
17. Li J., A. Shah, 2003. ROS Generation by Nonphagocytic NADPH Oxidase: Potential Relevance in Diabetic Nephropathy. J Am Soc Nephrol, 14: S221-S226.

18. Brown G. C., V. Borutaite, 2006. Interactions between nitric oxide, oxygen, reactive oxygen species and reactive nitrogen species. Biochem Soc Trans, 34(Pt 5) : 953-956

19. Storz P, 2006. Reactive oxygen species-mediated mitochondria-to-nucleus signaling: a key to aging and radical-caused diseases. Sci STKE, 2006 (332) re3.

20. Chen Q., E. J. Vazquez, S. Moghaddas, C. L. Hoppel, E. J. Lesnefsky, 2003, Production of reactive oxygen species by mitochondria: central role of complex III. J Biol Chem, 278(38):3602736031.

21. Kudin A.P., N.Y. Bimpong-Buta, S. Vielhaber, C. E. Elger, W. S. Kunz, 2004. Characterization of superoxide-producing sites in isolated brain mitochondria. J Biol Chem, 279(6): 4127-4135.

22. G. A. Martens, Y. Cai, S. Hinke, G. Stange, M. Van de Casteele, D. Pipeleers, 2005. Glucose suppresses superoxide generation in metabolically responsive pancreatic beta cells. J Biol Chem, 280(21):20389-20396.

23. Sethuraman M., N. Clavreul, H. Huang, M. E. McComb, C. E. Costello, R. A. Cohen, 2007. Quantification of oxidative posttranslational modifications of cysteine thiols of p21ras associated with redox modulation of activity using isotope-coded affinity tags and mass spectrometry. Free Radic Biol Med, 42(6):823-829.

24. Pacher P., J. S. Beckman, L. Liaudet, 2007. Nitric oxide and peroxynitrite in health and disease. Physiol Rev, 87(1):315-424.

25. Schrader M., H. D. Fahimi, 2006. Peroxisomes and oxidative stress. Biochim Biophys Acta, 1763(12):1755-1766.

26. Coon M. J., 2003. Multiple oxidants and multiple mechanisms in cytochrome P450 catalysis. Biochem Biophys Res Commun, 312(1):163-168.

27. S. J. Hewett, M. G. Espey, T. F. Uliasz, D. A. Wink., 2005. Neurotoxicity of nitroxyl: insights into $\mathrm{HNO}$ and NO biochemical imbalance. Free Radic Biol Med, 39 (11): 1478-14788.

28. Cross J. V., D. J. Templeton, 2006. Regulation of signal transduction through protein cysteine oxidation. Antioxid Redox Signal, 8(9-10):18191827. 
29. Berndt C. , C. H. Lillig, A. Holmgren, 2007. Thiolbased mechanisms of the thioredoxin and glutaredoxin systems: implications for diseases in the cardiovascular system. Am J Physiol Heart Circ Physiol. 292(3):H1227-236.

30. Nakamura H., 2005. Thioredoxin and its related molecules: update. Antioxidant Redox Signal, 7(56):823-828.

31. Holmgren A., C. Johansson, C. Berndt, M. E. Lonn, C. Hudemann, C.H. Lillig, 2005. Thiol redox control via thioredoxin and glutaredoxin systems. Biochem Soc Trans, 33(Pt 6):1375-1377.

32. A. A. Turanov, D. Su, V. N. Gladyshev, 2006. Characterization of alternative cytosolic forms and cellular targets of mouse mitochondrial thioredoxin reductase. J Biol Chem, 281(32): 22953-22963.

33. Liu Y., W. Min. 2002. Thioredoxin promotes ASK1 ubiquitination and degradation to inhibit ASK1-mediated apoptosis in a redox activityindependent manner. Circ Res, 90 (12): 1259-1266.

34. Wang Y., G. W. De Keulenaer, R. T. Lee, 2002. Vitamin D (3)-up-regulated protein-1 is a stressresponsive gene that regulates cardiomyocyte viability through interaction with thioredoxin, J Biol Chem, 277(29):26496-26500.

35. Wiesel P., L. C. Foster, A. Pellacani, M. D. Layne, C. M. Hsieh, G. S. Huggins, P. Strauss, S. F. Yet, M. A. Perrella, 2000. Thioredoxin facilitates the induction of heme oxygenase-1 in response to inflammatory mediators. J Biol Chem, 275 (32): 24840-24846.

36. Lundberg M., C. Johansson, J. Chandra, M. Enoksson, G. Jacobsson, J. Ljung, M. Johansson, A. Holmgren, 2001. Cloning and expression of a novel human glutaredoxin (Grx2) with mitochondrial and nuclear isoforms, $\mathrm{J}$ Biol Chem. 276(28): 26269-26275.

37. S. M. Beer, E. R. Taylor, S. E. Brown, C. C. Dahm, N. J. Costa, M. J. Runswick, M. P. Murphy, 2004. Glutaredoxin 2 catalyzes the reversible oxidation and glutathionylation of mitochondrial membrane thiol proteins: implications for mitochondrial redox regulation and antioxidant DEFENSE. J Biol Chem, 279(46): 47939-47951.

38. Landar A., J. Y. Oh, N. M. Giles, A. Isom, M. Kirk, S. Barnes, V. M. Darley-Usmar, 2006. A sensitive method for the quantitative measurement of protein thiol modification in response to oxidative stress. Free Radic Biol Med, 40(3):459468.
39. Nystrom T., 2005. Role of oxidative carbonylation in protein quality control and senescence.EMBO J, 24(7):1311-1317.

40. Whittier J. E., Y. Xiong, M. C. Rechsteiner, T. C. Squier, 2004. Hsp90 enhances degradation of oxidized calmodulin by the $20 \mathrm{~S}$ proteasome, J Biol Chem, 279 (44): 46135-46142.

41. Yan B., H. Wang, Z. N. Rabbani, Y. Zhao, W. Li, Y. Yuan, F. Li, M. W. Dewhirst, C. Y. Li, 2006. Tumor necrosis factor-alpha is a potent endogenous mutagen that promotes cellular transformation. Cancer Res, 66(24):11565-11570.

42. Terada L. S., 2006. Specificity in reactive oxidant signaling: think globally, act locally. J. Cell Biol, 174:615-623.

43. Rehmann H., A. Wittinghofer, J. L. Bos, 2007. Capturing cyclic nucleotides in action: snapshots from crystallographic studies. Nat Rev Mol Cell Biol, 8(1):63-73.

44. Hool L.C., B. Corry, 2007. Redox control of calcium channels: from mechanisms to therapeutic opportunities. Antioxid Redox Signal, 9(4):409435.

45. C. M. Spickett, A. R. Pitt, N. Morrice, W. Kolch, 2006. Proteomic analysis of phosphorylation, oxidation and nitrosylation in signal transduction. Biochim Biophys Acta, 1764(12):1823-1841.

46. Bossis G., F. Melchior, 2006. Regulation of SUMOylation by reversible oxidation of SUMO conjugating enzymes. Mol Cell, 21(3):349-357.

47. Bubici C., S. Papa, K. Dean, G. Franzoso, 2006. Mutual cross-talk between reactive oxygen species and nuclear factor-kappa B: molecular basis and biological significance. Oncogene, 25(51):67316748.

48. Han M. J., B. Y. Kim, S. O. Yoon, A. S. Chung, 2003. Cell proliferation induced by reactive oxygen species is mediated via mitogen-activated protein kinase in Chinese hamster lung fibroblast (V79) cells. Mol Cells, 15(1):94-101.

49. Ross B., R. B. Mikkelsen, P. Wardman, 2003. Biological chemistry of reactive oxygen and nitrogen and radiation-induced signal transduction mechanisms. Oncogene, 22: 5734-5754.

50. O'Brian C. A., F. Chu, 2005. Post-translational disulfide modifications in cell signaling--role of inter-protein, intra-protein, S-glutathionyl, and Scysteaminyl disulfide modifications in signal transmission. Free Radic Res, 39(5):471-480. 
51. Mikkelsen R. B., P Wardman. 2003. Biological chemistry of reactive oxygen and nitrogen and radiation-induced signal transduction mechanisms. Oncogene, 22(37): 5734-5754.

52. Zima A. V., L. A. Blatter. 2006. Redox regulation of cardiac calcium channels and transporters. Cardiovasc Res, 71(2):310-321.

53. Cheong E., V. Tumbev, J. Abramson, G. Salama, D.A. Stoyanovsky, 2005. Nitroxyl triggers Ca2+ release from skeletal and cardiac sarcoplasmic reticulum by oxidizing ryanodine receptors. Cell Calcium, 37(1):87-96.

54. Martinez-Moreno M., A. Alvarez-Barrientos, F. Roncal, J. P. Albar, F. Gavilanes, S. Lamas, I. Rodriguez-Crespo, 2005. Direct interaction between the reductase domain of endothelial nitric oxide synthase and the ryanodine receptor. FEBS Lett, 579(14):3159-3163.

55. Cohen R. A., T. Adachi, 2006. Nitric-oxideinduced vasodilatation: regulation by physiologic s-glutathiolation and pathologic oxidation of the sarcoplasmic endoplasmic reticulum calcium ATPase. Trends Cardiovasc Med, 16(4):109-114.

56. Mirzaei H., F. Regnier, 2006. Protein-RNA crosslinking in the ribosomes of yeast under oxidative stress. J Proteome Res, 5(12):3249-3259.

57. Golstein P., G. Kroemer, 2007. Cell death by necrosis: towards a molecular definition. Trends Biochem Sci, 32(1):37-43.

58. Hajnoczky G., J. B. Hoek, 2007. Cell signaling. Mitochondrial longevity pathways. Science. 315(5812):607-609.

59. Forrester M.T., J. S. Stamler, 2007. A classification scheme for redox-based modifications of proteins. Am J Respir Cell Mol Biol, 36(2):135-137.

60. Wang X. W., Y. C. Liou, B. Ho, J. L. Ding, 2007. An evolutionarily conserved $16-\mathrm{kDa}$ thioredoxinrelated protein is an antioxidant which regulates the NF-kappaB signaling pathway. Free Radic Biol Med, 42 (2):247-259.

61. Weber H., S. Huhns, L. Jonas, G. Sparmann, M. Bastian, P. Schuff-Werner, 2007. Hydrogen peroxide-induced activation of defense mechanisms against oxidative stress in rat pancreatic acinar AR42J cells. Free Radic Biol Med, 42(6):830-841.

62. Smith P. S., W. Zhao, D. R. Spitz, M. E. Robbins, 2007. Inhibiting catalase activity sensitizes $36 \mathrm{~B} 10$ rat glioma cells to oxidative stress. Free Radic Biol Med, 42(6):787-797.
63. Li L., W. Shoji, H. Takano, N. Nishimura, Y. Aoki, R. Takahashi, S. Goto, T. Kaifu, T. Takai, M. Obinata, 2007. Increased susceptibility of MER5 (peroxiredoxin III) knockout mice to LPS-induced oxidative stress. Biochem Biophys Res Commun, Feb 12 [Epub].

64. Noctor G., R. De Paepe, C. H. Foyer, 2007. Mitochondrial redox biology and homeostasis in plants. Trends Plant Sci, Feb 9 [Epub].

65. Henderson B. C., S. C. Tyagi. 2006. Oxidative mechanism and homeostasis of proteinase/ antiproteinase in congestive heart failure. $\mathrm{J}$ Mol Cell Cardiol, 41(6): 959-962.

66. Filomeni G., M. R. Ciriolo, 2006. Redox control of apoptosis: an update. Antioxid Redox Signal. 8(1112):2187-2192.

67. Jacob C., I. Knight, P. G. Winyard, 2006. Aspects of the biological redox chemistry of cysteine: from simple redox responses to sophisticated signalling pathways. Biol Chem, 387(10-11):1385-1397.

68. Szegezdi E., S. E. Logue, A. M. Gorman, A. Samali, 2006. Mediators of endoplasmic reticulum stress-induced apoptosis, EMBO Rep, 7(9):880885.

69. Ogura Y., F. S. Sutterwala, R. A. Flavell, 2006. The inflammasome: first line of the immune response to cell stress. Cell, 126(4):659-662.

70. Ischiropoulos H., J. S. Beckman, 2003. Oxidative stress and nitration in neurodegeneration: cause, effect, or association? J Clin Invest, 111(2):163169.

71. Buttke T. M., P. A. Sandstrom. 1994. Oxidative stress as a mediator of apoptosis. Immunol Today, 15(1):7-10.

72. Mao W., C. Iwai, P. C. Keng, R. Vulapalli, C. S. Liang, 2006. Norepinephrine-induced oxidative stress causes PC-12 cell apoptosis by both endoplasmic reticulum stress and mitochondrial intrinsic pathway: inhibition of phosphatidylinositol 3-kinase survival pathway. Am J Physiol Cell Physiol, 290(5):C1373-1384.

73. Liu Y., G. L. Borchert, A. Surazynski, C. A. Hu, J. M. Phang, 2006. Proline oxidase activates both intrinsic and extrinsic pathways for apoptosis: the role of ROS/superoxides, NFAT and MEK/ERK signaling. Oncogene, 14;25(41):5640-5647.

74. Taddei M. L., M. Parri, T. Mello, A. Catalano, A. D. Levine, G. Raugei, G. Ramponi, P. Chiarugi, 2007. Integrin-mediated cell adhesion and spreading engage different sources of reactive oxygen species. Antioxid Redox Signal. 9(4):46981. 
75. Filomeni G., M. R. Ciriolo, 2006. Redox control of apoptosis: an update. Antioxid Redox Signal, 8(11-12):2187-2192.

76. Kajstura J., R. Bolli, E. H. Sonnenblick, P. Anversa, A. Leri, 2006. Cause of death: suicide? J Mol Cell Cardiol, 40(4):425-437.

77. Gonzalez A., M. A. Fortuno, R. Querejeta, S. Ravassa, B. Lopez, N. Lopez, J. Diez, 2003. Cardiomyocyte apoptosis in hypertensive cardiomyopathy. Cardiovasc Res, 59(3):549-562.

78. Han H., H. Long, H. Wang, J. Wang, Y. Zhang, Z. Wang, 2004. Progressive apoptotic cell death triggered by transient oxidative insult in $\mathrm{H} 9 \mathrm{c} 2$ rat ventricular cells: a novel pattern of apoptosis and the mechanisms. Am J Physiol Heart Circ Physiol, 286: H2169-2182.

79. Lin M. T., M. F. Beal, 2006. Mitochondrial dysfunction and oxidative stress in neurodegenerative diseases. Nature, 443 (7113): 787-795.

80. Hirotani S., K. Otsu, K. Nishida, Y. Higuchi, T. Morita, H. Nakayama, O. Yamaguchi, T. Mano, Y. Matsumura, H. Ueno, M. Tada, M. Hori, 2002. Involvement of nuclear factor- appaB and apoptosis signal-regulating kinase 1 in G-proteincoupled receptor agonist-induced cardiomyocyte hypertrophy. Circulation, 105: 509-515.

81. Pang J. J., R. K. Xu., X. B. Xu., J. M. Cao, C. Ni., W. L. Zhu, K. Asotra, M. C. Chen, C. Chen, 2004. Hexarelin protects rat cardiomyocytes from angiotensin II-induced apoptosis in vitro. Am J Physiol Heart Circ Physiol, 286: H1063-1069.

82. Ricci R., U. Eriksson, G. Y. Oudit, R. Eferl, A. Akhmedov, I. Sumara, G. Sumara, Z. Kassiri, J. P. David, L. Bakiri, B. Sasse, M. H. Idarraga, M. Rath, D. Kurz, H. C. Theussl, J. C. Perriard, P. Backx, J. M. Penninger, E. F. Wagner, 2005. Distinct functions of junD in cardiac hypertrophy and heart failure. Genes Dev, 208-213.
83. Kwon S. H., D. R. Pimentel, A. Remondino, D.B. Sawyer, W.S. Colucci, 2003. H2O2 regulates cardiac myocyte phenotype via concentrationdependent activation of distinct kinase pathways. J Mol Cell Cardiol, 35:615-621.

84. Clerk A, 2003. The radical balance between life and death. J. Mol Cell Cardiol, 35:599-602.

85. Gupta M. K., T. V. Neelakantan, M. Sanghamitra, R. K. Tyagi, A. Dinda, S. Maulik, C. K. Mukhopadhyay, S. K. Goswami, 2006. An assessment of the role of reactive oxygen species and redox signaling in norepinephrine-induced apoptosis and hypertrophy of $\mathrm{H} 9 \mathrm{c} 2$ cardiac myoblasts. Antioxid Redox Signal, 8(5-6):1081-93.

86. Frazier D. P., A. Wilson, C. J. Dougherty, H. Li, N. H. Bishopric, K. A. Webster, 2007. PKC-\& agr; and TAK-1 are Intermediates in the Activation of c-Jun N-terminal Kinase (JNK) by HypoxiaReoxygenation. Am J Physiol Heart Circ Physiol, [Epub].

87. Yaniv G., M. Shilkrut, S. Larisch, O. Binah, 2005. Hydrogen peroxide predisposes neonatal rat ventricular myocytes to Fas-mediated apoptosis. Biochem Biophys Res Commun, 336(3):740-746.

88. Kostin S., L. Pool, A. Elsasser, S. Hein, H. C. Drexler, E. Arnon, Y. Hayakawa, R. Zimmermann, E. Bauer, W. P. Klovekorn, J. Schaper, 2003. Myocytes die by multiple mechanisms in failing human hearts, Circ Res, 92(7):715-724. 